\title{
Enhancement of pair correlation in a one-dimensional hybridization model
}

\author{
Yupeng Wang \\ Institut für Physik, Universität Augsburg, D-86135 Augsburg, Germany \\ and Laboratory of Ultra-Low Temperature Physics, Chinese Academy of Sciences, P.O. Box 2711, Beijing 100080, \\ People's Republic of China \\ Jian-Hui Dai \\ Condensed Matter Section, International Center for Theoretical Physics, P.O. Box 586, Trieste 34100, Italy \\ $\mathrm{Fu}-\mathrm{Cho} \mathrm{Pu}$ \\ Department of Physics, Guangzhou Teacher College, Guangzhou 510400, People's Republic of China \\ and Institute of Physics, Chinese Academy of Sciences, Beijing 100080, People's Republic of China \\ Ulrich Eckern \\ Institut für Physik, Universität Augsburg, D-86135 Augsburg, Germany \\ (Received 7 July 1998; revised manuscript received 23 October 1998)
}

\begin{abstract}
We propose an integrable model of one-dimensional interacting electrons coupled with the local orbitals arranged periodically in the chain. Since the local orbitals are introduced in a way that double occupation is forbidden, the model keeps the main features of the periodic Anderson model with an interacting host. For an attractive interaction, it is found that the local orbitals enhance the effective mass of the Cooper-pair-like singlets and also the pair correlation in the ground state. However, the persistent current is depressed in this case. For the repulsive interaction case, the Hamiltonian is non-Hermitian but allows Cooper pair solutions with small momenta, which are induced by the hybridization between the extended states and the local orbitals. [S0163-1829(99)11207-4]
\end{abstract}

\section{INTRODUCTION}

Metallic compounds containing elements with partially filled $d$ shells or $f$ shells belong to the category of strongly correlated electrons. Two typical examples are the high- $T_{c}$ superconductors and the heavy fermion compounds in which spin fluctuations may play a central role. ${ }^{1,2}$ The normal state properties of heavy fermion compounds are characterized by a large Pauli susceptibility and specific heat as compared to conventional metals. Such phenomena are attributed to the large effective mass of the electrons near the Fermi surface. These anomalies are generally believed to be due to the formation of resonant states at the Fermi level, which is induced by the admixture of local $f$ orbitals and the conduction electrons, and therefore the systems are usually modeled by the periodic Anderson model or the Kondo lattice model in some limiting cases. One of the major mysteries of the heavy fermions is how superconductivity could be supported in a system with strong local moments. ${ }^{2}$ It is generally accepted that magnetic impurities in BCS superconductors break the time reversal symmetry and are unfavorable to the formation of Cooper pairs. Such a pair-breaking effect directly causes the reduction of the energy gap of the superconducting state and the transition temperature. ${ }^{3}$ However, the situation may be different in some strongly correlated electron systems, where the electrons from the same source could be responsible for both the superconductivity and the magnetism. ${ }^{1}$ How magnetism and superconductivity reconcile each other is still a hot topic in modern condensed matter physics and remains an open problem.
Based on the development of the strongly correlated electron systems and low-dimensional systems, many efforts have been made in recent years to understand how the magnetic impurities behave in a one-dimensional (1D) correlated host. ${ }^{4}$ Several integrable models were proposed ${ }^{5-7}$ to account for this problem and some novel features were found. In a recent paper, ${ }^{8}$ Schlottmann studied the attractive Hubbard model with a finite concentration of magnetic impurities. He found that the impurities generally weaken the binding energy of the singlet pairs and the spin gap could be closed above a critical concentration of impurities.

The quantum inverse scattering method (QISM) provides a powerful tool to construct integrable models in one dimension. ${ }^{9}$ In a lattice model, a local operator $L_{n, \tau}(\lambda)$ can be defined, which satisfies the following Yang-Baxter relation: ${ }^{10}$

$$
R_{\tau, \tau^{\prime}}(\lambda, \mu) L_{n, \tau}(\lambda) L_{n, \tau^{\prime}}(\mu)=L_{n, \tau^{\prime}}(\mu) L_{n, \tau}(\lambda) R_{\tau, \tau^{\prime}}(\lambda, \mu),
$$

where $L_{n, \tau}(\lambda)$ acts on the auxiliary space $V_{\tau}$ and the quantum space $V_{n}$, respectively, $\lambda$ and $\mu$ are the spectral parameters, and $R_{\tau, \tau^{\prime}}(\lambda, \mu)=L_{\tau, \tau^{\prime}}(\lambda-\mu)$ is a $c$-number matrix. Define the transition matrix $T_{\tau}(\lambda)$ as

$$
T_{\tau}(\lambda)=L_{1, \tau}(\lambda) \cdots L_{N, \tau}(\lambda),
$$

where $N$ is the site number of the lattice. From Eq. (1) we can easily show that

$$
R_{\tau, \tau^{\prime}}(\lambda-\mu) T_{\tau}(\lambda) T_{\tau^{\prime}}(\mu)=T_{\tau^{\prime}}(\mu) T_{\tau}(\lambda) R_{\tau, \tau^{\prime}}(\lambda-\mu) .
$$


Tracing $\tau$ and $\tau^{\prime}$ in the above equation we have

$$
\left[\operatorname{tr}_{\tau} T_{\tau}(\lambda), \operatorname{tr}_{\tau} T_{\tau}(\mu)\right]=0 .
$$

Suppose $\tau(\lambda) \equiv \operatorname{tr}_{\tau} T_{\tau}(\lambda)$ allows the following expansion:

$$
\tau(\lambda)=t_{0}+\frac{t_{1}}{\lambda}+\frac{t_{2}}{\lambda^{2}}+\cdots
$$

Since $\lambda$ and $\mu$ are arbitrary parameters, from Eq. (4) we obtain

$$
\left[t_{m}, t_{n}\right]=0, \quad m, n=0,1,2, \ldots
$$

Choosing one of the $t_{n}$ as the Hamiltonian of the system, from Eq. (6) we know that all $t_{m}$ are conserved quantities. Therefore, we can establish the common eigenstates of these quantities. Generally, the generating operators of these eigenstates are chosen from the off-diagonal elements of the matrix $T_{\tau}(\lambda)$. For the impurity models, the impurities are added by including some inhomogeneous vertex operators in the transition matrix, ${ }^{7}$ which satisfy the same Yang-Baxter relation as that of the host.

In this paper, we consider a model of 1D interacting electrons coupled to the local orbitals arranged periodically in the chain. Maximumly, only one electron can occupy a single local state. Therefore, the model preserves the main feature of a 1D periodic Anderson model in the limit $U$ $\rightarrow \infty$. The structure of the present paper is the following: In the subsequent section, the model Hamiltonian and its Bethe ansatz solution will be constructed based on the QISM. In Sec. III, we discuss the attractive interaction case. It is shown that the effective mass of the Cooper-pair-like singlets as well as the pair correlation in the ground state are enhanced by the local orbitals. In Sec. IV, we study the repulsive interaction case. Section $\mathrm{V}$ gives our concluding remarks.

\section{THE MODEL AND ITS BETHE ANSATZ}

In this paper, we construct an integrable model, which describes interacting conduction electrons in a continuum medium hybridizing with local orbitals. The atoms with local orbitals are arrayed periodically in the chain and the local states are described by the Hubbard operators $X_{\alpha, \beta}^{n}$ $\equiv\left|\alpha_{n}\right\rangle\left\langle\beta_{n}\right|\left(\alpha_{n}, \beta_{n}=0, \uparrow, \downarrow\right)$, with the constraint $X_{\uparrow \uparrow}^{n}+X_{\downarrow \downarrow}^{n}$ $+X_{00}^{n}=1$, which means double occupation of the same orbital is forbidden. Let us start with the following segment transition matrix:

$$
\partial_{x} T_{n+1}(\lambda \mid x, n a)=: L(\lambda, x) T_{n+1}(\lambda \mid x, n a):
$$

where $x \in[n a,(n+1) a)$; :: denotes the normal order of the fermions, $a$ is the space between two nearest local orbitals, and the $L$ operator is defined as

$$
L(\lambda, x)=\left(\begin{array}{ccc}
\frac{i}{2} \lambda & 0 & i \sqrt{g} c_{\uparrow}(x) \\
0 & \frac{i}{2} \lambda & i \sqrt{g} c_{\downarrow}(x) \\
-i \sqrt{g} c_{\uparrow}^{\dagger}(x) & -i \sqrt{g} c_{\downarrow}^{\dagger}(x) & -\frac{i}{2} \lambda
\end{array}\right),
$$

with $c_{\sigma}^{\dagger}(x)\left[c_{\sigma}(x)\right]$ the creation (annihilation) operator of the conduction electrons. Here, we take the boundary condition of $T_{n+1}(\lambda \mid x, n a)$ as

$$
T_{n+1}(\lambda \mid n a, n a) \equiv L_{n}(\lambda)=\left(\begin{array}{ccc}
a^{\prime}(\lambda)-b^{\prime}(\lambda) X_{\uparrow, \uparrow}^{n} & -b^{\prime}(\lambda) X_{\downarrow, \uparrow}^{n} & b^{\prime}(\lambda) X_{0, \uparrow}^{n} \\
-b^{\prime}(\lambda) X_{\uparrow, \downarrow}^{n} & a^{\prime}(\lambda)-b^{\prime}(\lambda) X_{\downarrow, \downarrow}^{n} & b^{\prime}(\lambda) X_{0, \downarrow}^{n} \\
b^{\prime}(\lambda) X_{0, \uparrow}^{n} & b^{\prime}(\lambda) X_{0, \downarrow}^{n} & a^{\prime}(\lambda)+b^{\prime}(\lambda) X_{0,0}^{n}
\end{array}\right),
$$

where $a^{\prime}(\lambda)=a(\lambda+i g / 2), b^{\prime}(\lambda)=b(\lambda+i g / 2)$, and

$$
a(\lambda)=\frac{\lambda}{\lambda-i g}, \quad b(\lambda)=\frac{-i g}{\lambda-i g}
$$

We remark that with a unit boundary condition, the transition matrix is just that of the $\delta$-potential Fermi gas model ${ }^{11}$ introduced by Yang. ${ }^{10}$ The nonunit boundary condition is very similar to the inhomogeneous $L$ operator in the lattice model. ${ }^{7}$ It is easy to show that the following Yang-Baxter relations hold:

$$
R(\lambda-\mu) L(\lambda, x) \otimes_{s} L(\mu, x)=L(\mu, x) \otimes_{s} L(\lambda, x) R(\lambda-\mu),
$$

$$
R(\lambda-\mu) L_{n}(\lambda) \otimes_{s} L_{n}(\mu)=L_{n}(\mu) \otimes_{s} L_{n}(\lambda) R(\lambda-\mu),
$$

where

$$
R(\lambda)=a(\lambda) P+b(\lambda),
$$

and $P$ is the fermion-fermion-boson (FFB) graded exchange operator acting on the direct product of the auxiliary spaces, $P_{a_{2} b_{2}}^{a_{1} b_{1}}=\delta_{a_{1} b_{2}} \delta_{a_{2} b_{1}}(-1)^{\epsilon_{b_{1}}} \epsilon_{b_{2}} ; \epsilon_{\uparrow, \downarrow}=1, \epsilon_{0}=0$ (for convenience, we put the subscripts $\uparrow, \downarrow$ and 0 as 1,2 , and 3, respectively); $\otimes_{s}$ denotes the direct product with FFB grading ${ }^{12,11}$

$$
\left(F \otimes_{s} G\right)_{c d}^{a b}=F_{a b} G_{c d}(-1)^{\epsilon_{c}\left(\epsilon_{a}+\epsilon_{b}\right)} .
$$

From Eqs. (9) and (10) we easily derive

$$
R(\lambda-\mu) T_{n}(\lambda) \otimes_{s} T_{n}(\mu)=T_{n}(\mu) \otimes_{s} T_{n}(\lambda) R(\lambda-\mu),
$$


with $T_{n}(\lambda) \equiv T_{n}(\lambda \mid n a, n a-a)$. Furthermore, the global transition matrix

$$
T(\lambda) \equiv T_{N}(\lambda) T_{N-1}(\lambda) \cdots T_{1}(\lambda)
$$

satisfies the same Yang-Baxter equation as the segment ones $T_{n}(\lambda)$,

$$
R(\lambda-\mu) T(\lambda) \otimes_{s} T(\mu)=T(\mu) \otimes_{s} T(\lambda) R(\lambda-\mu),
$$

where $N$ is the number of atoms with local orbital. Introduce the notation

$$
T(\lambda)=\left(\begin{array}{ccc}
A_{11}(\lambda) & A_{12}(\lambda) & B_{1}(\lambda) \\
A_{21}(\lambda) & A_{22}(\lambda) & B_{2}(\lambda) \\
C_{1}(\lambda) & C_{2}(\lambda) & D(\lambda)
\end{array}\right) .
$$

From Eq. (15) we have the following commutation relations:

$$
\begin{aligned}
& A_{a b}(\lambda) C_{c}(\mu)=(-1) \epsilon_{a} \epsilon_{p} \frac{r(\lambda-\mu)_{p b}^{d c}}{a(\lambda-\mu)} C_{p}(\mu) A_{a d}(\lambda) \\
& +\frac{b(\lambda-\mu)}{a(\lambda-\mu)} C_{b}(\lambda) A_{a c}(\mu), \\
& D(\lambda) C_{c}(\mu)=\frac{1}{a(\mu-\lambda)} C_{c}(\mu) D(\lambda) \\
& -\frac{b(\mu-\lambda)}{a(\mu-\lambda)} C_{c}(\lambda) D(\mu),
\end{aligned}
$$

$$
\begin{gathered}
\left.C_{a_{1}}\left(\lambda_{1}\right) C_{a_{2}}\left(\lambda_{2}\right)=r\left(\lambda_{1}-\lambda_{2}\right)\right)_{b_{2} a_{1}}^{b_{1} a_{1}} C_{b_{2}}\left(\lambda_{2}\right) C_{b_{1}}\left(\lambda_{1}\right), \\
{[\tau(\lambda), \tau(\mu)]=0,}
\end{gathered}
$$

where

$$
\tau(\lambda)=\operatorname{str} T(\lambda) \equiv-A_{11}(\lambda)-A_{22}(\lambda)+D(\lambda)
$$

and

$$
r(\lambda)_{c d}^{a b}=-b(\lambda) \delta_{a b} \delta_{c d}-a(\lambda) \delta_{a d} \delta_{b c} .
$$

It can be shown that $r(\lambda)$ satisfies the following YangBaxter relation:

$$
\begin{aligned}
& r(\lambda-\mu)_{a_{3} c_{3}}^{a_{2} c_{2}} r(\lambda){ }_{c_{2} d_{2}}^{a_{1} b_{1}} r(\mu){ }_{c_{3} b_{3}}^{d_{2} b_{2}} \\
& \quad=r(\mu)_{a_{2} c_{2}}^{a_{1} c_{1}} r(\lambda)_{a_{3} b_{3}}^{c_{2} d_{2}} r(\lambda-\mu)_{d_{2} b_{2}}^{c_{1} b_{1}} .
\end{aligned}
$$

From Eq. (19) we see that $\tau(\lambda)$ can be considered as a generator of an infinite number of conserved quantities. Choosing the vacuum state as

$$
c_{\sigma}(x)|0\rangle=X_{0, \sigma}^{n}|0\rangle=0
$$

we have
Therefore, $C_{a}(\lambda)$ can be treated as the creation operators of the eigenstates of $\tau(\lambda)$

$$
\left.\left|k_{1}, \ldots, k_{n}\right| F\right\rangle=C_{a_{1}}\left(k_{1}\right) C_{a_{2}}\left(k_{2}\right) \cdots C_{a_{n}}\left(k_{n}\right)|0\rangle F^{a_{n} \cdots a_{1}},
$$

where the indices $a_{j}$ run over the values 1,2 , and $F^{a_{n} \cdots a_{1}}$ is a function of the spectral parameters $k_{j}$. From the commutation relations (16) and (17) we have ( $n$ is the electron number)

$$
\begin{aligned}
& \left.D(\lambda)\left|k_{1}, \ldots, k_{n}\right| F\right\rangle \\
& \left.=e^{-i(\lambda / 2) L} \prod_{j=1}^{n} \frac{1}{a\left(k_{j}-\lambda\right)}\left|k_{1}, \ldots, k_{n}\right| F\right\rangle \\
& \quad+\sum_{l=1}^{n}\left(\bar{\Lambda}_{l}\right)_{a_{1} \cdots a_{n}}^{b_{1} \cdots b_{n}} C_{b_{l}}(\lambda) \prod_{j \neq l}^{n} C_{b_{j}}\left(k_{j}\right)|0\rangle F^{a_{n} \cdots a_{1}},
\end{aligned}
$$

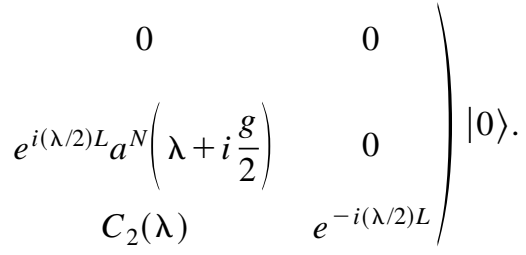

$$
\begin{aligned}
{\left[A_{11}(\lambda)\right.} & \left.\left.+A_{22}(\lambda)\right]\left|k_{1}, \ldots, k_{n}\right| F\right\rangle \\
= & -e^{i(\lambda / 2) L} a^{N}\left(\lambda+i \frac{g}{2}\right) \prod_{j=1}^{n} \frac{1}{a\left(\lambda-k_{j}\right)} \prod_{l=1}^{n} C_{b_{l}}\left(\lambda_{l}\right)|0\rangle \\
& \times\left[\tau^{(1)}(\lambda)\right]_{a_{1} \cdots a_{n}}^{b_{1} \cdots b_{n}} F^{a_{n} \cdots a_{1}}+\sum_{l=1}^{n}\left(\Lambda_{l}\right)_{a_{1} \cdots a_{n}}^{b_{1} \cdots b_{n}} C_{b_{l}}(\lambda) \\
& \times \prod_{j \neq l}^{n} C_{b_{j}}\left(k_{j}\right)|0\rangle F^{a_{n} \cdots a_{1}},
\end{aligned}
$$

where

$$
\tau^{(1)}(\lambda)=\operatorname{str}\left[T_{n}^{(1)}(\lambda)\right]=\operatorname{str}\left[L_{n}^{(1)}\left(\lambda-k_{n}\right) \cdots L_{1}^{(1)}\left(\lambda-k_{1}\right)\right]
$$

and 


$$
L_{k}^{(1)}(\lambda)=b(\lambda) P^{(1)}+a(\lambda)
$$

which fulfill the Yang-Baxter relation

$$
r(\lambda-\mu) L_{k}^{(1)}(\lambda) \otimes_{s} L_{k}^{(1)}(\mu)=L_{k}^{(1)}(\mu) \otimes_{s} L_{k}^{(1)}(\lambda) r(\lambda-\mu),
$$

with $\left(P^{(1)}\right)_{c d}^{a b}=-\delta_{a d} \delta_{b c}$ the $4 \times 4$ permutation matrix, and

$$
\begin{aligned}
& \left(\Lambda_{l} F\right)^{b_{1} \cdots b_{n}}=\frac{b\left(\lambda-k_{l}\right)}{a\left(\lambda-k_{l}\right)} \prod_{i \neq l}^{n} \frac{1}{a\left(k_{l}-k_{i}\right)} a^{N}\left(k_{l}+i \frac{g}{2}\right) \\
& \times e^{i\left(k_{l} / 2\right) L} F^{a_{n} \cdots a_{l} b_{l-1} \cdots b_{1}(-1)^{l+1}} \\
& \times L_{n}^{(1)}\left(k_{l}-k_{n}\right)_{b_{n} a_{n}}^{b_{l} d_{n-1}} \\
& \times L_{n-1}^{(1)}\left(k_{l}-k_{n-1}\right)_{b_{n-1} a_{n-1}}^{d_{n-3} d_{n-3}} \ldots \\
& \times L_{l+1}\left(k_{l}-k_{l+1}\right)_{b_{l+1} a_{l+1} a_{l}}, \\
& \left(\bar{\Lambda}_{l} F\right)^{b_{1} \cdots b_{n}}=S\left(k_{l}\right)_{a_{1} \cdots a_{l}}^{b_{1} \cdots b_{l}} F^{b_{n} \cdots b_{l+1} a_{l} \cdots a_{1}} \\
& \times\left[-\frac{b\left(k_{l}-\lambda\right)}{a\left(k_{l}-\lambda\right)}\right] e^{-i\left(k_{l} / 2\right) L} \prod_{i \neq l}^{n} \frac{1}{a\left(k_{i}-k_{l}\right)},
\end{aligned}
$$

where

$$
S\left(k_{l}\right)_{a_{1} \cdots a_{l}}^{b_{1} \cdots b_{l}}=r\left(k_{l-1}-k_{l}\right)_{c_{l-1} a_{l-1}}^{b_{l-1} a_{l}} \cdots r\left(k_{1}-k_{l}\right)_{b_{l} a_{1}}^{b_{1} c_{2}} .
$$

To obtain the Bethe ansatz equations (BAE) and the eigenvalues of $\tau(\lambda)$, we solve first the following eigenvalue problem:

$$
\tau^{(1)}(\lambda) F=e(\lambda) F .
$$

From Eq. (29) we know

$$
r(\lambda-\mu) T_{n}^{(1)}(\lambda) \otimes_{s} T_{n}^{(1)}(\mu)=T_{n}^{(1)}(\mu) \otimes_{s} T_{n}^{(1)}(\lambda) r(\lambda-\mu) .
$$

Introduce the notation

$$
T_{n}^{(1)}(\lambda)=\left(\begin{array}{ll}
A^{(1)}(\lambda) & B^{(1)}(\lambda) \\
C^{(1)}(\lambda) & D^{(1)}(\lambda)
\end{array}\right)
$$

then we find from Eq. (34)

$$
\begin{aligned}
D^{(1)}(\lambda) C^{(1)}(\mu)= & \frac{1}{a(\lambda-\mu)} C^{(1)}(\mu) D^{(1)}(\lambda) \\
& +\frac{b(\mu-\lambda)}{a(\mu-\lambda)} C^{(1)}(\lambda) D^{(1)}(\mu), \\
A^{(1)}(\lambda) C^{(1)}(\mu)= & \frac{1}{a(\mu-\lambda)} C^{(1)}(\mu) A^{(1)}(\lambda) \\
& +\frac{b(\lambda-\mu)}{a(\lambda-\mu)} C^{(1)}(\lambda) A^{(1)}(\mu),
\end{aligned}
$$

$$
C^{(1)}(\lambda) C^{(1)}(\mu)=C^{(1)}(\mu) C^{(1)}(\lambda),
$$

$$
\left[\tau^{(1)}(\lambda), \tau^{(1)}(\mu)\right]=0 \text {. }
$$

Define the pseudovacuum $|0\rangle^{(1)}$ as $B^{(1)}(\lambda)|0\rangle^{(1)}=0$. The eigenstates of $\tau^{(1)}(\lambda)$ can be written as

$$
\left|\mu_{1}, \ldots, \mu_{M}\right\rangle=\prod_{\alpha=1}^{M} C^{(1)}\left(\mu_{\alpha}\right)|0\rangle^{(1)} .
$$

Applying $\tau^{(1)}(\lambda)=-A^{(1)}(\lambda)-D^{(1)}(\lambda)$ on Eq. (38), we have

$$
\begin{aligned}
e(\lambda)= & -\left[\prod_{\alpha=1}^{M} \frac{1}{a\left(\lambda-\mu_{\alpha}\right)} \prod_{j=1}^{n} \frac{a\left(\lambda-k_{j}\right)}{a\left(k_{j}-\lambda\right)}\right. \\
& \left.+\prod_{\alpha=1}^{M} \frac{1}{a\left(\mu_{\alpha}-\lambda\right)} \prod_{j=1}^{n} a\left(\lambda-k_{j}\right)\right],
\end{aligned}
$$

and the cancellation of the unwanted terms gives the Bethe ansatz equation

$$
\prod_{i=1}^{n} a\left(k_{i}-\mu_{\alpha}\right)=\prod_{\alpha \neq \beta}^{M} \frac{a\left(\mu_{\beta}-\mu_{\alpha}\right)}{a\left(\mu_{\alpha}-\mu_{\beta}\right)} .
$$

To ensure Eq. (24) to be an eigenstate of $\tau(\lambda)$, the unwanted terms in Eqs. (25) and (26) must cancel, i.e.,

$$
\left[-\left(\Lambda_{l}\right)_{a_{1} \cdots a_{n}}^{b_{1} \cdots b_{n}}+\left(\bar{\Lambda}_{l}\right)_{a_{1} \cdots a_{n}}^{b_{1} \cdots b_{n}}\right] F^{a_{n} \cdots a_{1}}=0 .
$$

This gives

$$
a^{N}\left(k_{l}+i \frac{g}{2}\right) e^{i k_{l} L}=\prod_{\alpha=1}^{M} a\left(k_{l}-\mu_{\alpha}\right) .
$$

[For the detailed derivation of Eqs. (30), (31), and (42), we refer the readers to the Appendix B of Ref. 12, since the algebraic structure of the present model is almost the same as theirs.] The eigenvalue of $\tau(\lambda)$ reads

$$
\begin{aligned}
\nu\left(\lambda,\left\{k_{j}\right\},\left\{\mu_{\alpha}\right\}\right)= & a^{N}\left(\lambda+i \frac{g}{2}\right) e^{i(\lambda / 2) L} \prod_{j=1}^{n} \frac{1}{a\left(\lambda-k_{j}\right)} e(\lambda) \\
& +e^{-i(\lambda / 2) L} \prod_{j=1}^{n} \frac{1}{a\left(k_{j}-\lambda\right)},
\end{aligned}
$$

where $M$ is the number of electrons with down spin. Putting $\mu_{\alpha}=\lambda_{\alpha}-i g / 2$, the Bethe ansatz Eqs. (40) and (42) are reduced to

$$
e^{i k_{j} N a}\left(\frac{k_{j}+\frac{i}{2} g}{k_{j}-\frac{i}{2} g}\right)^{N}=\prod_{\alpha=1}^{M} \frac{k_{j}-\lambda_{\alpha}+\frac{i}{2} g}{k_{j}-\lambda_{\alpha}-\frac{i}{2} g},
$$




$$
\prod_{j=1}^{n} \frac{\lambda_{\alpha}-k_{j}-\frac{i}{2} g}{\lambda_{\alpha}-k_{j}+\frac{i}{2} g}=-\prod_{\beta=1}^{M} \frac{\lambda_{\alpha}-\lambda_{\beta}-i g}{\lambda_{\alpha}-\lambda_{\beta}+i g},
$$

and the eigenvalue of $\tau(\lambda)$ can be written clearly as

$$
\begin{aligned}
\nu\left(\lambda,\left\{k_{j}\right\},\left\{\lambda_{\alpha}\right\}\right)= & e^{-i(\lambda / 2) L} \prod_{j=1}^{n}\left(1+\frac{i g}{\lambda-k_{j}}\right) \\
& -e^{i(\lambda / 2) L}\left(\frac{\left.\lambda+i \frac{g}{2}\right)^{N}}{\lambda-i \frac{g}{2}}\right)^{N} \\
& \times\left\{\prod_{\alpha=1}^{M} \frac{\lambda-\lambda_{\alpha}-i \frac{g}{2}}{\lambda-\lambda_{\alpha}+i \frac{T^{2}}{2}} \prod_{j=1}^{n}\left(1+\frac{i g}{\lambda-k_{j}}\right)\right. \\
& \left.+\prod_{\alpha=1}^{M}\left(1+\frac{i g}{\lambda-\lambda_{\alpha}+i \frac{g}{2}}\right)\right\}
\end{aligned}
$$

Now we turn to the construction of the model Hamiltonian. For $\lambda \rightarrow \infty$ in the upper-half complex plane, we have the following asymptotic expansion:

$$
\ln \left[\tau(\lambda) e^{i(\lambda / 2) L}\right]=1+i g\left\{\frac{C_{1}}{\lambda}+\frac{C_{2}}{\lambda^{2}}+\frac{C_{3}}{\lambda^{3}}+\cdots\right\} .
$$

From the commutation relation (19) we know

$$
\left[C_{m}, C_{n}\right]=0, \quad m, n=1,2,3, \ldots
$$

In principle, we have freedom to choose a Hamiltonian from the conserved quantities $\left\{C_{n}\right\}$. In this paper, we define the Hamiltonian as

$$
H=C_{3}+i g C_{2}-\frac{1}{2} g^{2} C_{1} \text {. }
$$

For unit boundary condition $T_{n+1}(n a, n a)=1$, Eq. (49) leads via Neumann expansion to ${ }^{11}$

$$
\begin{aligned}
H_{0}= & \sum_{\sigma} \int \frac{\partial c_{\sigma}^{\dagger}(x)}{\partial x} \frac{\partial c_{\sigma}(x)}{\partial x} \\
& +2 g \int c_{\uparrow}^{\dagger}(x) c_{\downarrow}^{\dagger}(x) c_{\downarrow}(x) c_{\uparrow}(x) d x,
\end{aligned}
$$

which is nothing but the Hamiltonian of a 1D electron gas with $\delta$-potential interactions. With the local orbitals, Eq. (49) reads

$$
H=H_{0}+H_{I},
$$

where $H_{I}$ is very complicated, containing a hybridization term $\sum_{n, k} V_{n, k} c_{\sigma}^{\dagger}(k) X_{0, \sigma}^{n}+$ H.c., a correlation term $\Sigma_{n, \sigma, \sigma^{\prime}} U_{\sigma, \sigma^{\prime}} c_{\sigma^{\dagger}}^{\dagger}(n a) c_{\sigma}(n a) X_{\sigma^{\prime}, \sigma^{\prime}}^{n}$, and other irrelevant terms that often appear in integrable impurity models. ${ }^{7}$ Comparing Eqs. (47) and (46), we easily obtain the spectrum of the Hamiltonian to be given by

$$
E=\sum_{j=1}^{n} k_{j}^{2}
$$

\section{ATTRACTIVE INTERACTION}

We discuss first the attractive interaction, i.e., $g<0$ case. Without the local orbitals, the ground state is a Fermi sea filled by Cooper-pair-like bound pairs. Now from Eqs. (44) and (45), we can show how the local levels behave in the ground state. Carefully checking the Bethe ansatz equations we find that the Cooper-pair states described by

$$
k_{\alpha}^{ \pm}=\lambda_{\alpha} \pm \frac{i}{2} g
$$

are still possible solutions in the thermodynamic limit $L$ $\rightarrow \infty$, despite the existence of the local orbitals, where $\lambda_{\alpha}$ are real. To study the stability of these pair states, we consider a reference state, i.e., all the $n$ electrons form $n / 2$ pairs. In this case, the Bethe ansatz equations are reduced to

$$
e^{2 i \lambda_{\alpha} N a}\left(\frac{\lambda_{\alpha}-i|g|}{\lambda_{\alpha}+i|g|}\right)^{N}=\prod_{\beta=1}^{n / 2} \frac{\lambda_{\alpha}-\lambda_{\beta}-i|g|}{\lambda_{\alpha}-\lambda_{\beta}+i|g|},
$$

and the energy of this state is given by

$$
E=\sum_{\alpha=1}^{n / 2} 2 \lambda_{\alpha}^{2}-\frac{n g^{2}}{4}
$$

Taking the logarithm of Eq. (54) we obtain

$$
2 \lambda_{\alpha}+\frac{1}{a} \theta\left(\lambda_{\alpha}\right)=\frac{2 \pi I_{\alpha}}{L}+\sum_{\beta=1}^{n / 2} \theta\left(\lambda_{\alpha}-\lambda_{\beta}\right),
$$

where $\theta(x)=2 \tan ^{-1}(x /|g|)$ and $I_{\alpha}$ are integers or half integers depending on the parity of $N-n / 2$. Notice that each $I_{\alpha}$ corresponds to a pair state, and they must be different from each other due to the exclusion principle. The minimum state (with lowest energy) is thus described by a sequence of $\left\{I_{\alpha}\right\}=\{-(n / 2-1) / 2, \ldots,(n / 2-1) / 2\}$. In the thermodynamic limit $\left(n / L\right.$ finite), the distribution of $\lambda_{\alpha}$ can be described by a density function

$$
\rho\left(\lambda_{\alpha}\right)=\lim _{L \rightarrow \infty} \frac{1}{\left(\lambda_{\alpha+1}-\lambda_{\alpha}\right) L}
$$

which satisfies

$$
\rho(\lambda)=\frac{1}{\pi}+\frac{1}{a} f(\lambda)-\int_{-\lambda_{F}}^{\lambda_{F}} f\left(\lambda-\lambda^{\prime}\right) \rho\left(\lambda^{\prime}\right) d \lambda^{\prime}
$$

where the cutoff $\lambda_{F}$ is given by

$$
\int_{-\lambda_{F}}^{\lambda_{F}} \rho(\lambda) d \lambda=\frac{n}{2 L}
$$

and $f(\lambda)=|g| / \pi\left(\lambda^{2}+g^{2}\right)$. Without the local orbitals, the density distribution of $\lambda$ in the ground state takes the form 


$$
\begin{gathered}
\rho_{0}(\lambda)=\frac{1}{\pi}-\int_{-\lambda_{F}^{0}}^{\lambda_{F}^{0}} f\left(\lambda-\lambda^{\prime}\right) \rho_{0}\left(\lambda^{\prime}\right) d \lambda^{\prime}, \\
\int_{-\lambda_{F}^{0}}^{\lambda_{F}^{0}} \rho_{0}(\lambda) d \lambda=\frac{n}{2 L} .
\end{gathered}
$$

Comparing Eqs. (58) and (60) we can readily read off $\lambda_{F}$ $<\lambda_{F}^{0}$. That means the effective Fermi energy is reduced by the hybridization relative to that of the homogeneous system, a typical heavy fermion behavior. ${ }^{2}$ Now we consider the density of states at the Fermi surface. The energy density of the minimum state (relative to the chemical potential) can be written as

$$
E / L=\int_{-\lambda_{F}}^{\lambda_{F}}\left(2 \lambda^{2}-\frac{g^{2}}{2}-2 \mu\right) \rho(\lambda) d \lambda,
$$

where $\mu$ is the chemical potential. Substituting Eq. (58) into Eq. (62), we obtain

$$
E / L=\int_{-\lambda_{F}}^{\lambda_{F}}\left[\frac{1}{\pi}+\frac{1}{a} f(\lambda)\right] \epsilon(\lambda) d \lambda,
$$

where $\epsilon(\lambda)$ is the dressed energy ${ }^{13}$ of the Cooper pairs,

$$
\epsilon(\lambda)=2 \lambda^{2}-\frac{g^{2}}{2}-2 \mu-\int_{-\lambda_{F}}^{\lambda_{F}} f\left(\lambda-\lambda^{\prime}\right) \epsilon\left(\lambda^{\prime}\right) d \lambda^{\prime} .
$$

Consider then a particle-hole excitation relative to the minimum state. The density of the hole and that of the excited particle can be expressed as

$$
\rho_{h}(\lambda)=\frac{1}{L} \delta\left(\lambda-\lambda_{h}\right), \quad \rho_{p}(\lambda)=\frac{1}{L} \delta\left(\lambda-\lambda_{p}\right)
$$

where $\lambda_{h}$ and $\lambda_{p}$ are the centers of the hole and the particle, respectively. In addition, such an excitation induces the back flow of the Fermi sea, i.e., $\delta \rho(\lambda)$. From the BAE we have

$$
\begin{aligned}
\rho(\lambda)+\delta \rho(\lambda)= & \frac{1}{\pi}+\frac{1}{a} f(\lambda)+\rho_{h}(\lambda)-\rho_{p}(\lambda)-\int f\left(\lambda-\lambda^{\prime}\right) \\
& \times\left[\rho\left(\lambda^{\prime}\right)+\delta \rho\left(\lambda^{\prime}\right)+\rho_{p}\left(\lambda^{\prime}\right)-\rho_{h}\left(\lambda^{\prime}\right)\right] d \lambda^{\prime}
\end{aligned}
$$

With Eq. (58) we find

$$
\begin{aligned}
\delta \rho(\lambda)= & \rho_{h}(\lambda)-\rho_{p}(\lambda)+\frac{1}{L}\left[f\left(\lambda-\lambda_{p}\right)-f\left(\lambda-\lambda_{h}\right)\right] \\
& -\int_{-\lambda_{F}}^{\lambda_{F}} f\left(\lambda-\lambda^{\prime}\right) \delta \rho\left(\lambda^{\prime}\right) d \lambda^{\prime} .
\end{aligned}
$$

The excitation energy reads

$$
\delta E=L \int_{-\lambda_{F}}^{\lambda_{F}} 2 \lambda^{2} \delta \rho(\lambda) d \lambda-2 \lambda_{h}^{2}+2 \lambda_{p}^{2} .
$$

Substituting Eq. (67) into Eq. (68) we readily obtain

$$
\delta E=\epsilon\left(\lambda_{p}\right)-\epsilon\left(\lambda_{h}\right) .
$$

Therefore, $\epsilon(\lambda)$ can be treated as the quasiparticle energy of the elementary excitations. The excitation of breaking a Cooper pair can be treated in a similar manner. Such an excitation can be described by a $\lambda$ hole in the Fermi sea and two real $k$ modes $k_{1}, k_{2}$ above the Fermi level. In this case, the excitation energy is

$$
\delta E=-\epsilon\left(\lambda_{h}\right)-2 \mu+k_{1}^{2}+k_{2}^{2}+\frac{g^{2}}{2} .
$$

Notice that the dressed energy has the properties $\epsilon\left( \pm \lambda_{F}\right)$ $=0, \epsilon(\lambda)<0$ for $|\lambda|<\lambda_{F}$ and $\epsilon(\lambda)>0$ for $|\lambda|>\lambda_{F}$. Concerning the excitation near the Fermi surface, i.e., $\lambda_{h} \rightarrow \lambda_{F}$, $k_{1}^{2}, k_{2}^{2} \rightarrow \mu$, from Eq. (70) we readily obtain that there is a finite gap $g^{2} / 2$ to break a Cooper pair at the Fermi surface. The energy gap seems not to be changed by the local orbitals. The minimum state we introduced is thus the absolute ground state of the system for a given $n$. From the BAE (56) we see that the "quasiparticle" (Cooper pair) momenta can be defined as $p\left(\lambda_{\alpha}\right)=2 \pi I_{\alpha} / L$; then in our case $p^{\prime}(\lambda)$ $=2 \pi \rho(\lambda)$. The density of states at the Fermi surface is thus

$$
N\left(\lambda_{F}\right)=\left.\frac{1}{\pi} \frac{d p(\lambda)}{d \epsilon(\lambda)}\right|_{\lambda=\lambda_{F}}=\frac{2 \rho\left(\lambda_{F}\right)}{\epsilon^{\prime}\left(\lambda_{F}\right)} \equiv \frac{1}{\pi v},
$$

where $v$ is the sound velocity. ${ }^{9,13,14}$ Since $\rho\left(\lambda_{F}\right)>\rho_{0}\left(\lambda_{F}^{0}\right)$ and $\epsilon^{\prime}\left(\lambda_{F}\right)$ is an increasing function of $\lambda_{F}$, as can be shown from Eq. (64), we deduce that the density of states is enlarged by the local orbitals. This is not very strange because the Fermi sphere is compressed.

At present, we see that the local orbitals cannot destroy the Cooper-pair state completely. However, it is still not clear whether the local orbitals weaken the pair correlation or enhance it. To answer this question, let us consider the stiffness constant $K$, which measures the nonuniversal exponents of a variety of correlation functions in one dimension. ${ }^{14}$ For the integrable systems, $K=Z^{2}(\lambda)$, and the dressed charge function $Z(\lambda)$ (Ref. 13) in our case satisfies

$$
Z(\lambda)=1-\int_{-\lambda_{F}}^{\lambda_{F}} f\left(\lambda-\lambda^{\prime}\right) Z\left(\lambda^{\prime}\right) d \lambda^{\prime}
$$

Notice that the local orbitals do not change the form of $Z(\lambda)$ but the value of the cutoff $\lambda_{F}$. Easily we can show $d Z(\lambda) / d \lambda_{F}<0$, which means $Z(\lambda)$ is a monotonically decreasing function of $\lambda_{F}$. The stiffness constant $K$ is therefore also monotonically decreasing with $\lambda_{F}$. As stated in earlier publications, ${ }^{15}$ the gapless $1 \mathrm{D}$ quantum system is conformally invariant at zero temperature and the nonuniversal exponents of the correlation functions can be derived from the finite-size corrections of the energy spectrum: ${ }^{16,13} \delta E$ $=2 \pi v x_{\nu} / L$, where $x_{\nu}$ is the scaling dimension (one-half the critical exponent) of the relevant operator. In our case, the spin excitations have a finite gap while the charge excitations are gapless. Therefore, the charge sector is conformally invariant at zero temperature and the asymptotic long-distance superconducting correlators can be derived from the finitesize correction of the ground-state energy. ${ }^{13,14,16}$ Notice the pair operator $c_{\downarrow}^{\dagger}(x) c_{\uparrow}^{\dagger}(x)$ induces a pair-number change by 
one. The energy change induced by this operator can be calculated by following the standard method introduced in Ref. 13 as

$$
\delta E=E_{n / 2+1}^{g}-E_{n / 2}^{g}-2 \mu=4 \frac{\partial \mu}{\partial n}=\frac{\pi v}{2 K L},
$$

where $E_{m}^{g}$ is the ground-state energy with $m$ Cooper pairs. Hence, the pair correlator reads

$$
\begin{gathered}
\left\langle c_{\uparrow}(x) c_{\downarrow}(x) c_{\downarrow}^{\dagger}(0) c_{\uparrow}^{\dagger}(0)\right\rangle \sim x^{-\theta}, \\
\theta=1 / 2 K .
\end{gathered}
$$

Since $\theta<\theta_{0}$ (where $\theta_{0}$ is the corresponding exponent of the homogeneous system), we conclude that the local orbitals enhance the superconducting correlations. To see this clearly, let us consider the tunneling of the Cooper pairs through an impurity. The leading tunneling current through the impurity is

$$
\begin{gathered}
J(x, y) \sim-i\left[c_{\downarrow}^{\dagger}(x) c_{\uparrow}^{\dagger}(x) c_{\uparrow}(y) c_{\downarrow}(y)-\text { H.c. }\right], \\
x \sim 0^{-}, \quad y \sim 0^{+},
\end{gathered}
$$

where we have put the impurity at the origin. From the boundary conformal field theory ${ }^{17}$ we have the time correlator of $J$,

$$
\langle[J(t), J(0)]\rangle \sim t^{-4 \theta} .
$$

The tunneling conductance can be derived from the Kubo's formula $^{17}$ as

$$
\sigma(T) \sim T^{2 K^{-1}-2} .
$$

That means the local orbitals enhance the low-temperature tunneling conductance, which provides another evidence for the enhancement of the superconducting correlation at low temperatures. The Drude weight $D$, which measures the persistent current in one dimension, ${ }^{18}$ can also be derived exactly for the present model: $D=K v$. Thus we can deduce that $D$ is reduced by the local orbitals. That means the local orbitals suppress the persistent current.

\section{REPULSIVE INTERACTION}

Now we turn to the repulsive case $(g>0)$ where the Hamiltonian defined in Eq. (49) is non-Hermitian. We remark the study on non-Hermitian systems has drawn considerable attention recently for the applications in a variety of physical situations such as delocalization in disordered systems, ${ }^{19}$ quantum chaos, ${ }^{20}$ population biology in random media with convection, ${ }^{21}$ and metal-insulator transitions driven by an imaginary vector potential. ${ }^{22}$ The spectrum of a non-Hermitian Hamiltonian generally falls in the complex plane. ${ }^{23}$ In our case, from the Bethe ansatz Eq. (44) we can see that the solutions of $k_{j}$ are classified into two types: (i) $\left|\phi\left(k_{j}\right)\right|=1$; (ii) $\left|\phi\left(k_{j}\right)\right| \neq 1$, where

$$
\phi(k)=e^{i k a} \frac{k+\frac{i}{2} g}{k-\frac{i}{2} g} .
$$

For the Hermitian Hamiltonian, case (i) represents the real modes and case (ii) denotes the string solutions (Cooper pairs in the present model). Both of these two types of modes lead to real eigenvalues of the Hamiltonian. When $g>0$ in our case, the Hamiltonian contains a non-Hermitian hybridization term and a Hermitian term, which is nothing but the Hamiltonian of the repulsive $\delta$-potential Fermi gas. ${ }^{11}$ Even for $|\phi(k)|=1$, the Bethe ansatz equations have complex solutions. A typical solution is the imaginary mode $k=i \kappa$ with $\kappa a=\ln [|\kappa+g / 2| /|\kappa-g / 2|$ in the thermodynamic limit. An interesting feature is that the Bethe ansatz equations allow Cooper-pair solutions. For $\left|\phi\left(k_{j}\right)\right|>1$, the left-hand side of Eq. (44) is divergent in the thermodynamic limit, while for $\left|\phi\left(k_{j}\right)\right|<1$ it tends to zero. Therefore, the pair solutions (53) are allowed. However, there is a constraint for the real part of the pair solutions. The condition $|\phi(k)| \neq 1$ hints to

$$
e^{-(g / 2) a}\left|1+\frac{i g}{\lambda}\right|>1,
$$

which gives the critical value of the real part of the pair solutions as

$$
\lambda_{c}=\frac{g}{\sqrt{e^{g a}-1}} .
$$

The Cooper pair solution can exist only in the region $-\lambda_{c}$ $<\lambda<\lambda_{c}$. For the complex solutions in case (i), $|\operatorname{Im} k|$ $>|g| / 2$ when $|\operatorname{Re} k|<\lambda_{c}$. Hence the ground state consists of these modes and there is no Cooper pair in the ground state. Even so, we can see the local orbitals do cause some fluctuation towards the formation of Cooper pairs, since without these orbitals, the system does not have any bound state of electrons.

\section{CONCLUSION}

In conclusion, we propose an integrable hybridization model for a 1D correlated electron system. We remark, though the local orbitals are introduced periodically, the problem is still at the level of a single impurity since there is no correlation among the local states. In fact, the Bethe ansatz Eqs. (44) and (45) do not depend on the distribution of the local orbitals in real space. This remains a shortcoming of integrable models with many impurities, since the impurities introduced in such a way are completely transparent to the host electrons; only the forward scattering is included. Even so, a finite concentration of impurities enhances the superconducting correlation in our model. It seems that our result contradicts that of Ref. 8. The difference comes from (i) in our model, the binding energy is independent of the momenta of the pairs while in the attractive Hubbard model, it depends on the pair momenta ( $\Lambda$ in Ref. 8); (ii) there is no $E_{\text {imp }}$ in the formal expression of the eigenenergy (52), even though the local orbitals change the distribution of $\left\{k_{j}\right\}$; (iii) in our model, the density of electrons is not changed by the local orbitals. This corresponds to the hybridization configurations of $f^{0} \rightleftharpoons f^{1}$ for the local orbitals with a configuration $f^{0}$ in the atomic limit. We remark that impurities with higher spin and finite momenta can also be introduced in our model by a similar procedure. In this case, the spin momenta are 
split into two classes. One associated with the pair states (53) and the other representing the dynamics of the remaining spin degrees of freedom of the local orbitals. The latter excitations are always gapless as in the spin chains, and the spin excitations breaking a pair still have a finite gap.

\section{ACKNOWLEDGMENTS}

One of the authors (Y.W.) acknowledges the financial support of the Alexander von Humboldt-Stiftung and the China National Foundation for Natural Science.
${ }^{1}$ P.W. Anderson, Science 235, 1196 (1987); F.C. Zhang and T.M. Rice, Phys. Rev. B 37, 3759 (1988).

${ }^{2}$ For reviews, see D.W. Hess, P.S. Riseborough, and J.L. Smith, Encyclopedia of Applied Physics, edited by G.L. Trigg (VCH Publishers Inc., New York, 1991), Vol. 7, p. 435; N. Grewe and F. Steglich, Handbook on Physics and Chemistry of Rare Earths, edited by K.A. Gschneider, Jr. and L.L. Erying (Elsevier, Amsterdam, 1991), Vol. 14, p. 343; P.A. Lee, T.M. Rice, J.W. Serene, L.J. Sham, and J.W. Wilkins, Comments Condens. Matter Phys. 12, 99 (1986); H. Heffner and M.R. Norman, Comments Condens. Matter Phys. 17, 361 (1996).

${ }^{3}$ A.A. Abrikosov and L.P. Gor'kov, Zh. Éksp. Teor. Fiz. 39, 1781 (1960) [Sov. Phys. JETP 12, 1243 (1961)].

${ }^{4}$ D.H. Lee and J. Toner, Phys. Rev. Lett. 69, 3378 (1992); A. Frusaki and N. Nagaosa, ibid. 72, 892 (1994); P. Frödjh and H. Johannesson, ibid. 75, 300 (1995).

${ }^{5}$ Y. Wang, J. Dai, Z. Hu, and F.-C. Pu, Phys. Rev. Lett. 79, 1901 (1997); Y. Wang and J. Voit, ibid. 77, 4934 (1996); 78, 3799E (1997).

${ }^{6}$ Y. Wang, Phys. Rev. B 56, 14045 (1997); see also H. Frahm and A.A. Zvyagin, J. Phys.: Condens. Matter 9, 9939 (1997).

${ }^{7}$ N. Andrei and H. Johannesson, Phys. Lett. 100A, 108 (1984); G. Bedürttig, F.H. Eßler, and H. Frahm, Phys. Rev. Lett. 77, 5098 (1996); P. Schlottmann, Int. J. Mod. Phys. B 11, 355 (1997); P. Schlottmann and A.A. Zvyagin, Phys. Rev. B 55, 5027 (1997); Nucl. Phys. B 501, 728 (1997); Phys. Rev. B 56, 13989 (1997); A.A. Zvyagin and P. Schlottmann, J. Phys.: Condens. Matter 9, 3543 (1997); Phys. Rev. B 56, 300 (1997).

${ }^{8}$ P. Schlottmann, Phys. Rev. B 57, 10638 (1998).
${ }^{9}$ For example, see V.E. Korepin, A.G. Izergin, and N.M. Bogoliubov, Quantum Inverse Scattering Method, Correlation Functions, and Algebraic Bethe Ansatz (Cambridge University Press, Cambridge 1993).

${ }^{10}$ C.N. Yang, Phys. Rev. Lett. 19, 1312 (1967); R.J. Baxter, Ann. Phys. (N.Y.) 70, 193 (1972).

${ }^{11}$ F.-C. Pu and B.-H. Zhao, Phys. Rev. D 30, 1387 (1984); Nucl. Phys. B 275, 77 (1986).

${ }^{12}$ F.H.L. Essler and V.E. Korepin, Phys. Rev. B 46, 9147 (1992).

${ }^{13}$ N.M. Bogoliubov, A.G. Izergin, and N.Yu Reshetikhin, J. Phys. A 20, 5361 (1987); A.G. Izergin, V.E. Korepin, and N.Yu Reshetikhin, ibid. 22, 2615 (1989).

${ }^{14}$ F.D.M. Haldane, J. Phys. C 14, 2585 (1981); for a review, see J. Voit, Rep. Prog. Phys. 57, 977 (1994).

${ }^{15}$ A.A. Belavin, A.M. Polyakov, and A.B. Zamolodchikov, Nucl. Phys. B 241, 333 (1984).

${ }^{16}$ J.L. Cardy, J. Phys. A 17, L385 (1984); Nucl. Phys. B 270, 186 (1984); P. Nightingale and H. Blöte, J. Phys. A 16, L657 (1983).

${ }^{17}$ Y. Wang, J. Voit, and F.-C. Pu, Phys. Rev. B 54, 8491 (1996); for a scaling description, see J.L. Kane and M.P.A. Fisher, Phys. Rev. Lett. 69, 1201 (1992); Phys. Rev. B 46, 15233 (1992).

${ }^{18}$ B.S. Shastry and B. Sutherland, Phys. Rev. Lett. 65, 243 (1990); B. Sutherland and B.S. Shastry, ibid. 65, 1833 (1990).

${ }^{19}$ N. Hatano and D.R. Nelson, Phys. Rev. Lett. 77, 570 (1996).

${ }^{20}$ K.B. Efetov, Phys. Rev. Lett. 79, 491 (1997).

${ }^{21}$ D.R. Nelson and N.M. Shnerb, Phys. Rev. E 58, 1383 (1998).

${ }^{22}$ T. Fukui and N. Kawakami, Phys. Rev. B 58, 16051 (1998); R.A. Lehrer and D.R. Nelson, Phys. Rev. B 58, 12385 (1998).

${ }^{23}$ I.Y. Goldsheid and B.A. Khoruzhenko, Phys. Rev. Lett. 80, 2897 (1998); N.M. Shnerb and D.R. Nelson, ibid. 80, 5172 (1998). 